\title{
Resonant states and classical damping
}

\author{
Dariusz Chruściński \\ Institute of Physics, Nicholas Copernicus University \\ ul. Grudziądzka 5/7, 87-100 Toruń, Poland \\ e-mail: darch@phys.uni.torun.pl
}

\begin{abstract}
Using Koopman's approach to classical dynamical systems we show that the classical damping may be interpreted as appearance of resonant states of the corresponding Koopman's operator. It turns out that simple classical damped systems give rise to discrete complex spectra. Therefore, the corresponding generalized eigenvectors may be interpreted as classical resonant states.
\end{abstract}

\section{Introduction}

It is widely believed that resonant states in quantum mechanics are responsible for the irreversible dynamics of physical systems (see e.g. [四). In this paper we address a similar problem in classical mechanics. Usually resonant states are eigenstates of the Hamiltonian corresponding to complex eigenvalues " $E+i \Gamma$ " with $E, \Gamma \in \mathbb{R} . E$ and $\Gamma$ are called resonance energy and resonance width, respectively. According to Breit-Wigner formula $1 / \Gamma$ measures the lifetime of the resonant state. Although quantum mechanics teaches us that a self-adjoint operator has real eigenvalues this is true only for proper eigenvectors, that is, eigenvectors belonging to the Hilbert space. It is well known that generalized eigenvectors (which do not belong to the Hilbert space) may correspond to complex (generalized) eigenvalues. The proper mathematical language to describe generalized eigenvectors is the rigged Hilbert space (or the Gelfand triplet) [2], [3]:

$$
D \subset \mathcal{H} \subset D^{*}
$$

where $D$ is a dense nuclear subspace in $\mathcal{H}$ and $D^{*}$ is a dual of $D$. Using convenient Dirac notation $\langle\phi \mid \psi\rangle$ to denote the action of $\psi \in D^{*}$ on a test function $\phi \in D$, we call an element $\psi$ a generalized eigenvector of a self-adjoint operator $A$ in $\mathcal{H}$ if

$$
\langle A \phi \mid \psi\rangle=\lambda\langle\phi \mid \psi\rangle
$$


for any test function $\phi \in D$.

Recently, it was observed by Kossakowski [4], [5] that quantization of classical damped systems leads immediately to resonant states. Let us recall that usually generalized eigenvectors correspond to continuous spectrum, e.g. plane waves for a free particle. Interestingly, studying quantized damped harmonic oscillator and the quantized toy model of the classical damped system described by

$$
\dot{x}=-\gamma x
$$

where $\gamma>0$ denotes a damping constant, Kossakowski found that both spectra are discrete and complex. In the present paper we show that the original classical damped systems may be treated in perfect analogy to their quantum counterparts. Moreover, the characteristic exponential decay

$$
x(t) \propto e^{-\gamma t}
$$

may be interpreted as appearance of a classical resonant state. The methods of Hilbert spaces are not reserved to quantum mechanics. As is well known classical Hamiltonian systems may be investigated in a very similar way. It was already observed by Koopman [6] that the Hamiltonian dynamics gives rise to one-parameter unitary group in the Hilbert space of square integrable function on the classical phase space (we review the Koopman's approach in Section 2.). Therefore, the spectrum of the corresponding self-adjoint generator should contain basic physical information about the classical system. It turns out that the corresponding Koopman's operators have very similar spectra as their quantum counterparts. Both spectra are discrete and complex. Their imaginary parts are responsible for the damping phenomena on a purely classical level.

\section{Koopman's approach}

Consider a classical Hamiltonian system defined on a symplectic manifold $(\mathcal{P}, \Omega)$. Recall, that $\Omega$ gives rise to the Poisson bracket in the space of functions on $\mathcal{P}$ :

$$
\{F, G\}:=\Omega\left(\mathcal{L}_{F}, \mathcal{L}_{G}\right)
$$

where $\mathcal{L}_{F}$ and $\mathcal{L}_{G}$ are Hamiltonian vector fields corresponding to $F$ and $G$, respectively. The classical phase space $\mathcal{P}$ carries the canonical volume form $d \mu$ which is the exterior product of $n$ copies of $\Omega($ where $2 n=\operatorname{dim} \mathcal{P})$ :

$$
d \mu:=\Omega \wedge \ldots \wedge \Omega
$$

One usually calls $d \mu$ the Liouville measure on $\mathcal{P}$. In local canonical coordinates $\left(x^{1}, \ldots, x^{n}, p_{1}, \ldots, p_{n}\right)$ on $\mathcal{P}$ one has $\left.d \mu=\prod_{i=1}^{n} d x^{i} d p_{i}\right)$. Define the Hilbert $L^{2}(\mathcal{P}, d \mu)$ space of square integrable function on $\mathcal{P}$ :

$$
L^{2}(\mathcal{P}, d \mu)=\left\{f:\left.\mathcal{P} \longrightarrow \mathbb{C}\left|\int\right| f\right|^{2} d \mu<\infty\right\}
$$


together with a Hermitian product:

$$
\langle f \mid g\rangle=\int \bar{f} g d \mu
$$

for any $f, g \in L^{2}(\mathcal{P}, d \mu)$. It was observed by Koopman [6] (see also [7] and [8] for the review of Koopman's approach) that a canonical transformation on $\mathcal{P}$, i.e. a map

$$
\varphi: \mathcal{P} \longrightarrow \mathcal{P}
$$

leaving a Poisson bracket invariant, gives rise to a unitary operator on $L^{2}(\mathcal{P}, d \mu)$ :

$$
U(f):=\varphi^{*} f=f \circ \varphi
$$

for any $f \in L^{2}(\mathcal{P}, d \mu)$. Indeed, $U$ is linear and maps $L^{2}(\mathcal{P}, d \mu)$ into itself. Moreover, $U$ is bijection, i.e. any function $g \in L^{2}(\mathcal{P}, d \mu)$ may be written as $g=U(f)$ for some $f \in L^{2}(\mathcal{P}, d \mu)$ - clearly, $f=g \circ \varphi^{-1}$. Finally, $U$ defines an isometry:

$$
\|U(f)\|^{2}=\int_{\mathcal{P}}|f(\varphi(x))|^{2} d \mu(x)=\int_{\mathcal{P}}|f(x)|^{2} d \mu(x)=\|f\|,
$$

due to the $\varphi$-invariance of $d \mu$.

Now, let $H \in C^{\infty}(\mathcal{P})$ denotes the Hamiltonian and let $\Phi_{t}$ stand for the corresponding Hamiltonian flow on $\mathcal{P}$, i.e.

$$
(x(t), p(t))=\Phi_{t}\left(x_{0}, p_{0}\right), \quad \text { for } t \in(a, b) .
$$

Clearly, for any $t \in(a, b), \Phi_{t}$ defines a canonical transformation on $\mathcal{P}$. Recall, that if $F$ is a function on $\mathcal{P}$, then the corresponding Hamiltonian vector field, denoted by $\mathcal{L}_{F}$, is defined by:

$$
\mathcal{L}_{F}(G):=\{G, F\}
$$

for any function $G$ on $\mathcal{P}$. It turns out 90 that if the Hamiltonian flow $\Phi_{t}$ is complete, that is, it is defined for all $t \in \mathbb{R}$, then

$$
U(t)(f)=f \circ \Phi_{t},
$$

defines a continuous one-parameter group of unitary transformation on $L^{2}(\mathcal{P}, d \mu)$. Moreover, one shows that

$$
U(t)=e^{\mathcal{L}_{H} t}
$$

and the Stone's theorem implies that $i \mathcal{L}_{H}$ is self-adjoint. 


\section{Examples of classical spectra}

To get a feeling how the classical spectra of $i \mathcal{L}_{H}$ may look like let us consider two simple examples. As a first example consider a free particle in $\mathbb{R}^{3}$. The corresponding phase space $\mathcal{P}=\mathbb{R}^{6}$ and the Hamiltonian is given by $H(\mathbf{x}, \mathbf{p})=$ $\mathbf{p}^{2} / 2 m$. Therefore, the Koopman's operator $i \mathcal{L}_{H}$ reads:

$$
i \mathcal{L}_{H}=\frac{i}{2 m} \mathbf{p} \cdot \nabla
$$

and it is evidently self-adjoint. The corresponding eigenvalue problem is easy to solve. One finds

$$
i \mathcal{L}_{H} f_{\mathbf{k}}(\mathbf{x}, \mathbf{p})=\frac{k^{2}}{2 m} f_{\mathbf{k}}(\mathbf{x}, \mathbf{p})
$$

where the eigenvector $f_{\mathbf{k}}(\mathbf{x}, \mathbf{p})$ is given by:

$$
f_{\mathbf{k}}(\mathbf{x}, \mathbf{p})=\delta(\mathbf{p}-\mathbf{k}) e^{-i \mathbf{k x}} .
$$

Hence, the spectrum is continuous, and the eigenvectors $f_{\mathbf{k}}$ do not belong to the Hilbert space $L^{2}\left(\mathbb{R}^{6}\right)$. However, we may consider the following Gelfand triplet

$$
\mathcal{S}\left(\mathbb{R}^{6}\right) \subset L^{2}\left(\mathbb{R}^{6}\right) \subset \mathcal{S}^{*}\left(\mathbb{R}^{6}\right)
$$

where by $\mathcal{S}\left(\mathbb{R}^{n}\right)$ the Schwartz space of rapidly decreasing functions on $\mathbb{R}^{n}$. The elements of the dual space $\mathcal{S}^{*}\left(\mathbb{R}^{n}\right)$ are called tempered distributions (see e.g. [10, 11]). Clearly, the generalized eigenvectors $f_{\mathbf{k}}$ are tempered distributions, i.e. elements from the dual space $\mathcal{S}^{*}\left(\mathbb{R}^{6}\right)$. Note, that

$$
\psi_{\mathbf{k}}(\mathbf{x})=\int d \mathbf{p} f_{\mathbf{k}}(\mathbf{x}, \mathbf{p})=e^{-i \mathbf{k x}}
$$

defines a generalized eigenvector, i.e. element from $\mathcal{S}^{*}\left(\mathbb{R}^{3}\right)$, for the corresponding quantum system with $\widehat{H}=-\left(\hbar^{2} / 2 m\right) \triangle$, that is,

$$
\widehat{H} \psi_{\mathbf{k}}=\frac{\hbar^{2} k^{2}}{2 m} \psi_{\mathbf{k}}
$$

Therefore, there is the direct correspondence between the spectrum of $\widehat{H}$ and that of $i \mathcal{L}_{H}$.

Our next example is a harmonic oscillator. In suitable coordinates the oscillator Hamiltonian is given by:

$$
H_{\mathrm{osc}}=\frac{\omega}{2}\left(x^{2}+p^{2}\right),
$$

and therefore, the corresponding Koopman's operator reads:

$$
i \mathcal{L}_{H_{\mathrm{osc}}}=i \omega\left(p \partial_{x}-x \partial_{p}\right)
$$


Clearly, the Hamiltonian flow $\Phi_{t}^{\text {osc }}$ is complete, and hence $i \mathcal{L}_{H_{\text {osc }}}$ defines a selfadjoint operator on $L^{2}\left(\mathbb{R}^{2}\right)$. Introducing complex coordinates $(z=x+i p, \bar{z}=$ $x-i p$ ) one obtains:

$$
H_{\mathrm{osc}}=\frac{\omega}{2}|z|^{2}
$$

and

$$
i \mathcal{L}_{H_{\mathrm{osc}}}=\omega(z \partial-\bar{z} \bar{\partial})
$$

where we use the standard notation: $\partial=\partial / \partial z$ and $\bar{\partial}=\partial / \partial \bar{z}$. One immediately finds the corresponding eigenvectors:

$$
(z \partial-\bar{z} \bar{\partial}) z^{n} \bar{z}^{m}=(n-m) z^{n} \bar{z}^{m}
$$

where $n, m$ are non-negative integers. Clearly the functions $z^{n} \bar{z}^{m}$ do not belong to $L^{2}\left(\mathbb{R}^{2}\right)$, however this deficiency may be easily cured. Observe, that any function of $|z|$ belongs to the kernel of $i \mathcal{L}_{H_{\mathrm{osc}}}$. Define the normalized functions:

$$
f_{n m}(z, \bar{z})=\frac{z^{n} \bar{z}^{m}}{\sqrt{\pi(n+m) !}} e^{-|z|^{2} / 2} .
$$

This normalization leads to the following formula:

$$
\left\langle f_{n m} \mid f_{k l}\right\rangle=\frac{(m+k) !}{\sqrt{(n+m) !(k+l) !}} \delta_{n-m, k-l} .
$$

Obviously

$$
i \mathcal{L}_{H_{\mathrm{osc}}} f_{n m}=\omega(m-n) f_{n m}
$$

that is, the spectrum of the Koopman's operator reads:

$$
\operatorname{Spec}\left(i \mathcal{L}_{H_{\mathrm{osc}}}\right)=\{\omega l \mid l \in \mathbb{Z}\}
$$

Let us compare the spectrum of $i \mathcal{L}_{H_{\text {osc }}}$ with the spectrum of $\widehat{H}_{\text {osc }}$ acting on $L^{2}(\mathbb{R})$. It is convenient to use the Bargmann representation [12], i.e. consider a Hilbert space of holomorphic functions $\psi=\psi(z)$ equipped with the following scalar product:

$$
\langle\psi \mid \phi\rangle_{\mathrm{B}}=\int \bar{\psi}(z) \phi(z) e^{-|z|^{2}} d z d \bar{z}
$$

Introducing standard creation and anihilation operators:

$$
\widehat{a}=\frac{\widehat{x}+i \widehat{p}}{\sqrt{2 \hbar}}, \quad \widehat{a}^{*}=\frac{\widehat{x}-i \widehat{p}}{\sqrt{2 \hbar}},
$$


one obtains

$$
\widehat{H}_{\mathrm{osc}}=\hbar \omega\left(a^{*} a+\frac{1}{2}\right)
$$

Moreover, the well known commutation relation

$$
\left[\widehat{a}, \widehat{a}^{*}\right]=1
$$

has the following holomorphic representation:

$$
\widehat{a} \psi(z)=\partial \psi(z) \quad \text { and } \quad \widehat{a}^{*} \psi(z)=z \psi(z),
$$

and therefore, due to (29), one obtains for the oscillator Hamiltonian:

$$
\widehat{H}_{\mathrm{osc}}=\hbar \omega\left(z \partial+\frac{1}{2}\right)
$$

Now the similarity between classical formula (21) and the quantum one (32) is evident. Recall that $\widehat{H}_{\text {osc }}$ acts on holomorphic functions, i.e. functions of $z$ only, whereas the classical counterpart $i \mathcal{L}_{H_{\text {osc }}}$ acts on functions depending both on $z$ and $\bar{z}$. Therefore, we have an additional term $\bar{z} \bar{\partial}$ in (21). The normalized eigenvectors of $\widehat{H}_{\text {osc }}$ are given by:

$$
\psi_{n}(z)=\frac{z^{n}}{\sqrt{\pi n !}}, \quad n=0,1,2, \ldots
$$

and hence

$$
\operatorname{Spec}\left(\widehat{H}_{\mathrm{osc}}\right)=\left\{\hbar \omega\left(n+\frac{1}{2}\right) \mid n=0,1,2, \ldots\right\}
$$

Note that the weight factor $e^{-|z|^{2}}$ present in the definition of the scalar product (27) is already contained in the classical eigenvectors $f_{n m}$, cf. (23). Clearly, we have the direct correspondence:

$$
f_{n 0} \longleftrightarrow \psi_{n} \quad \text { and } \quad f_{0 n} \longleftrightarrow \bar{\psi}_{n},
$$

and hence holomorphic $f_{n 0}$ are responsible for the positive part of the spectrum, whereas anti-holomorphic $f_{0 n}$ for the negative part.

\section{A toy model for a damped motion}

Now, let us turn to classical damped systems. Consider the simplest one:

$$
\dot{x}=-\gamma x
$$


Clearly this system is not Hamiltonian. However, it is well known (cf. e.g. [13]) that any dynamical system may be rewritten in a Hamiltonian form. Consider a dynamical system on $n$-dimensional configuration space $Q$ :

$$
\dot{x}=X(x),
$$

where $X$ is a vector field on $Q$. Now, define the following Hamiltonian on the cotangent bundle $\mathcal{P}=T^{*} Q$ :

$$
H\left(\alpha_{x}\right):=\alpha_{x}(X(x)),
$$

where $\alpha_{x} \in T_{x}^{*} Q$. Using canonical coordinates $\left(x^{1}, \ldots, x^{n}, p_{1}, \ldots, p_{n}\right)$ one obtains:

$$
H(x, p)=\sum_{k=1}^{n} p_{k} X^{k}(x),
$$

where $X^{k}$ are components of $X$ in the coordinate basis $\partial / \partial x^{k}$. The corresponding Hamilton equations take the following form:

$$
\begin{aligned}
& \dot{x}^{k}=\left\{x^{k}, H\right\}=X^{k}(x), \\
& \dot{p}_{k}=\left\{p_{k}, H\right\}=-\sum_{l=1}^{n} p_{l} \frac{\partial X^{l}(x)}{\partial x^{k}},
\end{aligned}
$$

for $k=1, \ldots, n$. In the above formulae $\{$,$\} denotes the canonical Poisson$ bracket on $T^{*} Q$ :

$$
\{F, G\}=\sum_{k=1}^{n}\left(\frac{\partial F}{\partial x^{k}} \frac{\partial G}{\partial p_{k}}-\frac{\partial G}{\partial x^{k}} \frac{\partial F}{\partial p_{k}}\right) .
$$

Clearly, the formulae (40) reproduce our initial dynamical system (37) on $Q$.

Let us apply the above procedure to the damped system (36). One obtains for the Hamiltonian

$$
H(x, p)=-\gamma x p,
$$

and hence the corresponding Hamilton equations

$$
\begin{aligned}
& \dot{x}=-\gamma x, \\
& \dot{p}=\gamma p,
\end{aligned}
$$

give rise to the following Hamiltonian flow on $\mathbb{R}^{2}$ :

$$
\Phi_{t}(x, p)=\left(e^{-\gamma t} x, e^{t \gamma} p\right) .
$$

Evidently, $\Phi_{t}$ is complete, and hence the Koopman's operator

$$
i \mathcal{L}_{H}=i \gamma\left(p \partial_{p}-x \partial_{x}\right) .
$$


is self-adjoint on $L^{2}\left(\mathbb{R}^{2}\right)$.1 Note that

$$
i \mathcal{L}_{H} g_{n m}=i \gamma(m-n) g_{n m}
$$

where

$$
g_{n m}(x, p)=x^{n} p^{m},
$$

and $n, m$ are non-negative integers. Interestingly, the generalized eigenvectors $g_{m n} \in \mathcal{S}^{*}\left(\mathbb{R}^{2}\right)$ correspond to purely imaginary discrete spectrum

$$
\operatorname{Spec}\left(i \mathcal{L}_{H}\right)=\{i \gamma k \mid k \in \mathbb{Z}\}
$$

Therefore, we call $g_{n m}$ the classical resonant states. Let us observe that

$$
g_{10}=x \quad \text { and } \quad g_{01}=p
$$

therefore

$$
i \mathcal{L}_{H} x=-i \gamma x \quad \text { and } \quad i \mathcal{L}_{H} p=i \gamma p
$$

that is, $x$ and $p$ are generalized eigenvectors of $i \mathcal{L}_{H}$ corresponding to imaginary eigenvalues $-i \gamma$ and $i \gamma$, respectively. This shows that the damping in a classical system $\dot{x}=-\gamma x$ corresponds to imaginary (generalized) eigenvalues of Koopman's Hamiltonian $i \mathcal{L}_{H}$ :

$$
U(t) x=e^{\mathcal{L}_{H} t} x=e^{-\gamma t} x \quad \text { and } \quad U(t) p=e^{\mathcal{L}_{H} t} p=e^{\gamma t} p
$$

or, in general

$$
U(t) f(x, p)=f\left(e^{-\gamma t} x, e^{\gamma t} p\right)
$$

There is a close relation between spectrum of a classical Koopman's operator $i \mathcal{L}_{H}$ and the spectrum of the corresponding quantum Hamiltonian [5]:

$$
\widehat{H}=-\frac{\gamma}{2}(\widehat{x} \widehat{p}+\widehat{p} \widehat{x})
$$

Let $\phi$ and $\psi$ be solutions of

$$
\widehat{x} \phi=0 \quad \text { and } \quad \widehat{p} \psi=0,
$$

${ }^{1}$ This may be easily checked by formal integration by parts:

$$
\begin{aligned}
\left\langle f, i \mathcal{L}_{H} g\right\rangle & =i \gamma \int \bar{f}\left(p \partial_{p}-x \partial_{x}\right) g d \mu \\
& =-i \gamma \int\left[p\left(\partial_{p} \bar{f}\right) g+f g-x\left(\partial_{x} \bar{f}\right) g-f g\right] d \mu=\left\langle i \mathcal{L}_{H} f, g\right\rangle,
\end{aligned}
$$

assuming that $f$ and $g$ vanish sufficiently fast for $|x|,|p| \longrightarrow \infty$. 
respectively. Clearly, up to non-important constants

$$
\phi(x)=\delta(x) \quad \text { and } \quad \psi(x)=1 .
$$

Note, that

$$
\widehat{H} \phi=-i \hbar \frac{\gamma}{2} \phi \quad \text { and } \quad \widehat{H} \psi=i \hbar \frac{\gamma}{2} \psi
$$

Now, define

$$
\phi_{n}:=\widehat{p}^{n} \phi \quad \text { and } \quad \psi_{n}:=\widehat{x}^{n} \psi
$$

with $n=1,2, \ldots$, i.e.

$$
\phi_{n}(x)=(-i \hbar)^{n} \delta^{(n)}(x) \quad \text { and } \quad \psi_{n}(x)=x^{n} .
$$

One easily finds that

$$
\widehat{H} \phi_{n}=-i \hbar \gamma\left(n+\frac{1}{2}\right) \phi_{n} \quad \text { and } \quad \widehat{H} \psi_{n}=+i \hbar \gamma\left(n+\frac{1}{2}\right) \psi_{n} .
$$

This shows that $\widehat{H}$ has a purely imaginary discrete spectrum:

$$
\operatorname{Spec}(\widehat{H})=\left\{i \hbar \gamma\left(k+\frac{1}{2}\right) \mid k \in \mathbb{Z}\right\}
$$

which is closely related to the classical spectrum (51). It should be stressed that (51) is not a classical limit of (63) (actually, the classical limit of (63) contains only zero eigenvalue). These two spectra corresponds to different operators defined in different spaces: $L^{2}\left(\mathbb{R}^{2}\right)$ and $L^{2}(\mathbb{R})$, respectively. Moreover, let us observe that there is direct correspondence between the generalized eigenvectors $g_{m n}$ and $\phi_{n}, \psi_{n}$ :

$$
x^{n}=g_{n 0}=\psi_{n}(x) \quad \text { and } \quad p^{n}=g_{0 n}=\int e^{i p x / \hbar} \phi_{n}(x) d x .
$$

Remark 1. There is a striking relation between classical oscillator spectrum (26) and the spectrum of damped system (51), i.e. a damped system has the same spectrum as a classical oscillator with imaginary frequency $\omega=i \gamma$. Let us observe that performing a canonical transformation $(x, p) \longrightarrow(X, P)$ :

$$
x=\frac{1}{\sqrt{2}}(X+P) \quad \text { and } \quad p=\frac{1}{\sqrt{2}}(X-P),
$$

one obtains

$$
H=-\gamma x p=\frac{\gamma}{2}\left(P^{2}-X^{2}\right)
$$


i.e. in the new variables $(X, P), H$ corresponds formally to the harmonic oscillator with $\omega= \pm i \gamma$. This correspondence may be easily seen if one applies the following linear operator [5]:

$$
V_{\lambda}:=e^{i \lambda \mathcal{L}_{X P}}=e^{i \lambda\left(P \partial_{P}-X \partial_{X}\right)}
$$

with $\lambda \in \mathbb{R}$. Clearly,

$$
V_{\lambda} X=e^{-i \lambda} X \quad \text { and } \quad V_{\lambda} P=e^{i \lambda} P
$$

and hence $V_{\lambda}$ defines a complex scaling. The above formulae imply:

$$
V_{\lambda}\left(P^{2}-X^{2}\right)=e^{2 i \lambda} P^{2}-e^{-2 i \lambda} X^{2}=e^{2 i \lambda}\left(P^{2}-e^{-4 i \lambda} X^{2}\right),
$$

so, if $\lambda= \pm \pi / 4$, then

$$
V_{ \pm \pi / 4}\left[\frac{\gamma}{2}\left(P^{2}-X^{2}\right)\right]= \pm \frac{i \gamma}{2}\left(P^{2}+X^{2}\right)
$$

that is, both systems are related by a complex scaling $V_{ \pm \pi / 4}$. Evidently, the same relation may be established between corresponding quantum systems and their spectra (34) and (63).

Remark 2. Let us note that if we replace (3) by a more general equation

$$
\dot{x}=-\gamma x^{n}
$$

with $n>1$, then the corresponding Hamiltonian system reproducing (71) is given by

$$
H=-\gamma x^{n} p
$$

The solution of (71) reads:

$$
x(t)=\frac{1}{[(n-1) \gamma t]^{1 / n-1}}+c,
$$

with $c=$ const., and evidently it is not complete. Therefore,

$$
U(t)=e^{t \mathcal{L}_{H}}
$$

is not defined for all $t \in \mathbb{R}$, and hence, Hamiltonian vector field

$$
i \mathcal{L}_{H}=i \gamma\left(n x^{n-1} p \partial_{p}-x^{n} \partial_{x}\right)
$$

does not define a self-adjoint operator for $n>1$. In particular for $n=2$ one obtains:

$$
e^{t \mathcal{L}_{H}} p=e^{2 \gamma x t} p
$$

and

$$
e^{t \mathcal{L}_{H}} x=\gamma x t+(\gamma x t)^{2}+(\gamma x t)^{3}+\ldots,
$$

which converges only for $|\gamma x t|<1$. Quantization of $H=x^{n} p$ leads to $\widehat{H} \propto$ $\widehat{x}^{n} \widehat{p}+\widehat{p} \widehat{x}^{n}$. It is well know (see e.g. [14) that this operator is not self-adjoint. 


\section{Damped harmonic oscillator}

Consider a damped harmonic oscillator defined by the following set of equations:

$$
\begin{aligned}
& \dot{x}=-\gamma x+\omega p, \\
& \dot{p}=-\gamma p-\omega x .
\end{aligned}
$$

Clearly this system is not Hamiltonian if $\gamma \neq 0$. Applying the procedure of [13 one arrives at the following Hamiltonian system on $\mathbb{R}^{4}$ (for the physical origin of this system cf. 《4):

$$
\begin{aligned}
& \dot{x}^{1}=\left\{x^{1}, H\right\}=-\gamma x^{1}+\omega x^{2} \\
& \dot{x}^{2}=\left\{x^{2}, H\right\}=-\omega x^{1}-\gamma x^{2} \\
& \dot{p}_{1}=\left\{p_{1}, H\right\}=+\gamma p_{1}+\omega p_{2} \\
& \dot{p}_{2}=\left\{p_{1}, H\right\}=-\omega p_{1}+\gamma p_{2}
\end{aligned}
$$

where we introduced $x^{1}=x$ and $x^{2}=p$. The Hamiltonian function is given by:

$$
H(x, p)=\omega\left(p_{1} x^{2}-p_{2} x^{1}\right)-\gamma\left(p_{1} x^{1}+p_{2} x^{2}\right) .
$$

Introducing complex variables:

$$
z_{1}=x^{1}+i x^{2} \quad \text { and } \quad z_{2}=i\left(p_{1}+i p_{2}\right)
$$

the Hamiltonian may be rewritten as follows:

$$
H\left(z_{1}, z_{2}, \bar{z}_{1}, \bar{z}_{2}\right)=\omega \operatorname{Re}\left(z_{1} \bar{z}_{2}\right)+\gamma \operatorname{Im}\left(z_{1} \bar{z}_{2}\right) .
$$

The corresponding Koopman's operator

$$
\begin{aligned}
i \mathcal{L}_{H} & =i \omega\left(x^{2} \partial_{x^{1}}-x^{1} \partial_{x^{2}}+p_{2} \partial_{p_{1}}-p_{1} \partial_{p_{2}}\right) \\
& +i \gamma\left(p_{1} \partial_{p_{1}}-x^{1} \partial_{x^{1}}+p_{2} \partial_{p_{2}}-x^{2} \partial_{x^{2}}\right)
\end{aligned}
$$

takes in the complex variables the following form:

$$
i \mathcal{L}_{H}=\left(\alpha z_{1} \partial_{1}-\bar{\alpha} \bar{z}_{1} \bar{\partial}_{1}\right)+\left(\bar{\alpha} z_{2} \partial_{2}-\alpha \bar{z}_{2} \bar{\partial}_{2}\right)
$$

where the complex parameter $\alpha$ is given by:

$$
\alpha=\omega-i \gamma
$$

The eigenvalue problem for $i \mathcal{L}_{H}$ is immediately solved by:

$$
i \mathcal{L}_{H} f_{n m k l}=\lambda_{n m k l} f_{n m k l}
$$

with

$$
f_{n m k l}\left(z_{1}, z_{2}\right)=z_{1}^{n} \bar{z}_{1}^{m} z_{2}^{k} \bar{z}_{2}^{l}
$$


and

$$
\begin{aligned}
\lambda_{n m k l} & =\alpha(n-l)-\bar{\alpha}(k-m) \\
& =\omega(n+k-m-l)-i \gamma(n+m-k-l) .
\end{aligned}
$$

Evidently $f_{n m k l} \notin L^{2}\left(\mathbb{R}^{4}\right)$. However, it is clear that the natural structure associated with the eigenvalue problem is the following Gelfand triplet:

$$
\mathcal{S}\left(\mathbb{R}^{4}\right) \subset L^{2}\left(\mathbb{R}^{4}\right) \subset \mathcal{S}^{*}\left(\mathbb{R}^{4}\right) .
$$

Now, the spectrum given by (92) is discrete and complex. Therefore, the classical resonant states $f_{n m k l}$ are responsible for the damping of harmonic oscillations. The unitary time evolution is given by:

$$
f_{t}\left(z_{1}, z_{2}, \bar{z}_{1}, \bar{z}_{2}\right)=e^{t \mathcal{L}_{H}} f\left(z_{1}, z_{2}, \bar{z}_{1}, \bar{z}_{2}\right)=f\left(e^{-i \alpha t} z_{1}, e^{-i \bar{\alpha} t} z_{2}, e^{i \bar{\alpha} t} \bar{z}_{1}, e^{i \alpha t} \bar{z}_{2}\right) .
$$

In particular

$$
z_{1}(t)=e^{-i \alpha t} z_{1}=e^{-i \omega t} e^{-\gamma t} z_{1}
$$

The quantized damped oscillator was analyzed in [4. Due to (84) the quantum Hamiltonian reads:

$$
\widehat{H}=\omega\left(\widehat{p}_{1} \widehat{x}_{2}-\widehat{p}_{2} \widehat{x}_{1}\right)-\frac{\gamma}{2}\left(\widehat{p}_{1} \widehat{x}_{1}+\widehat{x}_{1} \widehat{p}_{1}+\widehat{p}_{2} \widehat{x}_{2}+\widehat{x}_{2} \widehat{p}_{2}\right) .
$$

Introduce two sets of creaction and anihilation operators:

$$
\begin{aligned}
\widehat{a}_{1}=\frac{\widehat{x}_{1}+i \widehat{x}_{2}}{\sqrt{2 \hbar}}, & \widehat{a}_{1}^{*}=\frac{\widehat{x}_{1}-i \widehat{x}_{2}}{\sqrt{2 \hbar}}, \\
\widehat{a}_{2}=\frac{i \widehat{p}_{1}-\widehat{p}_{2}}{\sqrt{2 \hbar}}, & \widehat{a}_{2}^{*}=\frac{-i \widehat{p}_{1}-\widehat{p}_{2}}{\sqrt{2 \hbar}} .
\end{aligned}
$$

One finds that the following commutation relations hold:

$$
\begin{gathered}
{\left[\widehat{a}_{1}, \widehat{a}_{2}\right]=\left[\widehat{a}_{1}, \widehat{a}_{1}^{*}\right]=\left[\widehat{a}_{2}, \widehat{a}_{2}^{*}\right]=0,} \\
{\left[\widehat{a}_{1}, \widehat{a}_{2}^{*}\right]=\left[\widehat{a}_{2}, \widehat{a}_{1}^{*}\right]=1,}
\end{gathered}
$$

and hence, the Hamiltonian (96) may be rewritten as follows:

$$
\begin{aligned}
\widehat{H} & =\hbar\left(\alpha \widehat{a}_{2}^{*} \widehat{a}_{1}+\bar{\alpha} \widehat{a}_{1}^{*} \widehat{a}_{2}+\omega\right) \\
& =\hbar \alpha\left(\widehat{a}_{2}^{*} \widehat{a}_{1}+\frac{1}{2}\right)+\hbar \bar{\alpha}\left(\widehat{a}_{1}^{*} \widehat{a}_{2}+\frac{1}{2}\right) .
\end{aligned}
$$

with $\alpha$ defined in (89). Clearly, $\widehat{H}^{*}=\widehat{H}$. Commutation relations (99)-(100) may be represented in $C^{\infty}\left(\mathbb{R}^{2}\right)$ as follows $\llbracket$ :

$$
\begin{array}{lll}
\widehat{a}_{1}=\frac{1}{\sqrt{2}}\left(x+\partial_{y}\right), & \widehat{a}_{1}^{*}=\frac{1}{\sqrt{2}}\left(x-\partial_{y}\right), \\
\widehat{a}_{2}=\frac{1}{\sqrt{2}}\left(y+\partial_{x}\right), & \widehat{a}_{1}^{*}=\frac{1}{\sqrt{2}}\left(y-\partial_{x}\right) .
\end{array}
$$


Inserting (102)-(103) into (101) one obtains the following representation for $\widehat{H}$ :

$$
\widehat{H}=\hbar \omega\left(x y-\partial_{x y}^{2}\right)-i \hbar \gamma\left(y \partial_{y}-x \partial_{x}\right) .
$$

The spectrum oh $\widehat{H}$ is easy to find. One defines a "ground state" $\varphi_{00}$ to be the state satisfying

$$
\widehat{a}_{1} \varphi_{00}=\widehat{a}_{2} \varphi_{00}=0
$$

and

$$
\varphi_{n k}:=\left(\widehat{a}_{1}^{*}\right)^{n}\left(\widehat{a}_{2}^{*}\right)^{k} \varphi_{00}
$$

Now, it is easy to show that

$$
\widehat{H} \varphi_{n k}=\mu_{n k} \varphi_{n k}
$$

with

$$
\mu_{n k}=\hbar \alpha\left(n+\frac{1}{2}\right)+\hbar \bar{\alpha}\left(k+\frac{1}{2}\right)=\hbar \omega(n+k+1)-i \hbar \gamma(n-k) .
$$

Solving (105) one obtains (up to a non-important constant):

$$
\varphi_{00}(x, y)=e^{-x y}
$$

and hence the eigenstates $\varphi_{n k}$ do not belong to $L^{2}\left(\mathbb{R}^{2}\right)$.

Remark 3. Let us observe that commutation relations (99)-(100) may be represented in the space of holomorphic functions of two complex variables equipped with the following scalar product:

$$
\langle\psi \mid \phi\rangle=\int \bar{f}\left(z_{1}, z_{2}\right) g\left(z_{1}, z_{2}\right) e^{-z_{1} \bar{z}_{2}-\bar{z}_{1} z_{2}} d z_{1} d z_{2} d \bar{z}_{1} d \bar{z}_{2} .
$$

One has:

$$
\begin{array}{ll}
\widehat{a}_{1}=\partial_{1}, & \widehat{a}_{1}^{*}=z_{2}, \\
\widehat{a}_{2}=\partial_{2}, & \widehat{a}_{2}^{*}=z_{1} .
\end{array}
$$

Note, that contrary to (28) the above representation is not consistent with the corresponding Bargmann scalar product, that is, $\widehat{a}_{k}^{*}$ is not the adjoint of $\widehat{a}_{k}$ with respect to (27). Now, using (101) one obtains:

$$
\widehat{H}=\hbar\left(\alpha z_{1} \partial_{1}+\bar{\alpha} z_{2} \partial_{2}+\omega\right)
$$

which is the quantum analog of the Koopman's operator (88). The eigenvalue problem for (113) is solved by:

$$
\widehat{H} \psi_{n k}=\mu_{n k} \psi_{n k}
$$

with

$$
\psi_{n k}\left(z_{1}, z_{2}\right)=z_{1}^{n} z_{2}^{k},
$$

and $\mu_{n k}$ are given by(108). Clearly, holomorphic eigenvectors $f_{n 0 k 0}=\psi_{n k}$ and anti-holomorphic $f_{0 m 0 l}=\bar{\psi}_{m l}$. 


\section{Concluding remarks}

Let us observe that introducing polar coordinates $(r, \varphi)$ on $\mathbb{R}^{2}$, one finds that $i \mathcal{L}_{H_{\text {osc }}}$ defines a generator of $S O(2)$ rotation:

$$
i \mathcal{L}_{H_{\mathrm{osc}}}=i \omega \partial_{\varphi}
$$

Therefore, the eigenvalue problem for $i \mathcal{L}_{H_{\text {ocs }}}$ may be translated to the corresponding problem for $i \partial_{\varphi}$ with an obvious solution given by:

$$
i \omega \partial_{\varphi} e^{-i m \varphi}=m \omega e^{-i m \varphi}
$$

with $m \in \mathbb{Z}$. Hence, one recovers (26). Note, however, that we have reduced the problem from $L^{2}\left(\mathbb{R}^{2}\right)$ to $L^{2}\left(S^{1}\right)$, i.e. we consider a representation of $i \mathcal{L}_{H_{\text {osc }}}$ on a compact space $S^{1}$, which is a homogeneous space for $S O(2)$.

Now, let us turn to the damped system described by (66). Introducing hiperbolic coordinates:

$$
P=s \cosh \chi \quad \text { and } \quad X=s \sinh \chi,
$$

with $s \in(-\infty, \infty)$ and $\chi \in(-\infty, \infty)$ one easily finds that

$$
i \mathcal{L}_{H}=i \gamma \partial_{\chi}
$$

which defines a generator of $S O(1,1)$. Note however, that contrary to the generator of the compact group $S O(2)$, the corresponding generator of a non-compact $S O(1,1)$ has a continuous spectrum:

$$
i \gamma \partial_{\chi} e^{\beta \chi}=i \gamma \beta e^{\beta \chi}
$$

with $\beta \in \mathbb{R}$. But this is not what we have found in (51). This example shows that changing representation we may change the spectrum of the corresponding operator. Therefore, one has to be careful choosing the appropriate representation which has to be dictated by the physical problem in question. Actually, our problem is defined on $\mathbb{R}^{2}$, and hence, the corresponding Gelfand triplet $\mathcal{S}\left(\mathbb{R}^{2}\right) \subset$ $L^{2}\left(\mathbb{R}^{2}\right) \subset \mathcal{S}^{*}\left(\mathbb{R}^{2}\right)$ leads to the discrete spectrum.

Finally, let us note that the spectrum of a self-adjoint operator $A$ on a Hilbert space $\mathcal{H}$ does depend upon the space of test function $D$ in the definition of the Gelfand triplet (11). Consider for example a momentum operator $\widehat{p}=-i \partial_{x}$. Clearly $\widehat{p}$ has purely real eigenvalues $p \in \mathbb{R}$ corresponding to plane waves $\psi_{p}=$ $e^{i p x}$ :

$$
\widehat{p} \psi_{p}=p \psi_{p}
$$

\footnotetext{
${ }^{2}$ Clearly, this parameterization covers only the interior of the "light cone" $|P|>|X|$. To cover also the remaining region one chooses $P=s^{\prime} \sinh \chi^{\prime}$ and $X=s^{\prime} \cosh \chi^{\prime}$.
} 
but it has also complex eigenvalues $z$ :

$$
\widehat{p} \psi_{z}=z \psi_{z}
$$

with $\psi_{z}=e^{-i z x}$. Obviously neither $\psi_{k}$ nor $\psi_{z}$ belong to $L^{2}(\mathbb{R})$. Every student accepts " $p$ " but " $z$ " looks quite strange. However, mathematically they may be treated on the equal footing. It is evident that $\psi_{p}, \psi_{z} \in D^{*}(\mathbb{R})$, where $D(\mathbb{R})$ is the space of smooth function with compact supports equipped with the convex Schwartz topology (cf. e.g. [11]). Now, consider two subspaces $D_{+}(\mathbb{R})$ and $D_{-}(\mathbb{R})$ in $C^{\infty}(\mathbb{R})$ :

$$
\begin{aligned}
& D_{+}(\mathbb{R}):=\left\{\phi \in C^{\infty}(\mathbb{R}) \mid \operatorname{supp} \phi \subset(-\infty, c)\right\}, \\
& D_{-}(\mathbb{R}):=\left\{\phi \in C^{\infty}(\mathbb{R}) \mid \operatorname{supp} \phi \subset(c, \infty)\right\},
\end{aligned}
$$

for some $c \in \mathbb{R}$. Note, that

$$
\psi_{z} \in\left\{\begin{array}{ll}
D_{+}^{*}(\mathbb{R}), & \text { if } \operatorname{Im} z>0 \\
D_{-}^{*}(\mathbb{R}), & \text { if } \operatorname{Im} z<0
\end{array},\right.
$$

but $\psi_{p}$, which corresponds to $\operatorname{Im} z=0$, does not belong to $D_{ \pm}^{*}(\mathbb{R})$ for any $p$.

The author thanks Professor Andrzej Kossakowski for very interesting and

stimulating discussions. This work was partially supported by the Polish State Committee for Scientific Research (KBN) Grant no 2P03B01619.

\section{References}

[1] A. Bohm, H.-D. Doebner, P. Kielanowski, Irreversability and Causality, Semigroups and Rigged Hilbert Spaces, Lecture Notes in Physics 504, Springer, Berlin, 1998.

[2] I.M. Gelfand, N.J. Vilenkin, Generalized Functions, Vol. IV, Academic Press, New York, 1964.

[3] K. Maurin, General Eigenfunction Expansion and Unitary Representations of Topological Groups, PWN, Warszawa, 1968.

[4] A. Kossakowski, Open Sys. Information Dyn. 9 (2001) 1

[5] A. Kossakowski, private communication

[6] B. Koopman, Proc. Nat. Acad. Sci. 17 (1931) 315

[7] V.I. Arnold, A. Avez, Ergodic Problems of Classical Mechanics, W.A. Benjamin, Inc. New York, 1968 
[8] M. Reed, B. Simon, Methods of Modern Mathematical Physics. Vol. I. Functional Analysis, Academic, New York, 1974

[9] A. Povzner, Transl. Am. Math. Soc. 51 (1966) 189

[10] L. Schwartz, Théorie des distributions, vol. I, Hermann, Paris, 1957

[11] K. Yosida, Functional Analysis, Springer, Berlin, 1978

[12] V. Bargmann, Comm. Pure Appl. Math. 14 (1961) 187

[13] L.S. Pontriagin, V.G. Boltańskij, R.V. Gamkrelidze, E.F. Miscenko, The Mathematical Theory of Optimal Precesses, Wiley, New York, 1962

[14] H. Araki, Some Contact Points of Mathematics and Physics, preprint University of Bielefeld, 1976 